\title{
Error Control Method for Interactive Video Applications over Modern Communication Networks
}

\author{
Thuneibat S. A. ${ }^{1}$ \\ ${ }^{1}$ Department of Electrical Engineering, BAU, Jordan \\ Correspondence: Thuneibat S. A., Department of Electrical Engineering, BAU, Jordan. Tel: 962-775-613-054. \\ E-mail: Thuneibat@hotmail.com
}

Received: March 5, 2012 Accepted: March 28, 2012 Online Published: May 1, 2012

doi:10.5539/cis.v5n3p20

URL: http://dx.doi.org/10.5539/cis.v5n3p20

\begin{abstract}
In this paper we propose a new method of error control, suitable for interactive applications, through the organization of parallel connections and the modification of decision making device at receiver end. We developed an algorithm to control error in the information stream of interactive services and derived the procedure of the optimal decision making device at the receiver end, which realize error control of user information at the network layer. We used the simulation program MatLab and obtained the result curves which clearly show the advantageous benefits by sufficiently decrease the probability of error with increase the number of parallel connections.
\end{abstract}

Keywords: error control, video application, parallel connections, decision making device

\section{Introduction}

Modern communication networks based on multi protocol technologies are imposed large requirements such as: mobility services; quality of Service (QoS) parameters; ensuring security and integrity of transmitted information.

Most of the technologies implemented on the basis of modern communication networks can provide differentiated QoS, based on a particular method of classification of QoS requirements; IP is an example of providing such called "Best Effort" delivery with no guarantees of integrity and security.

The main criteria for the classification of QoS requirements using the following set of parameter, grouped under the probability and time characteristics:

- Bandwidth parameters: average, minimum and maximum data transfer rate

- Delay parameters: average and maximum delay values, as well as average and maximum jitter between packets of data

- Reliability of the transmission of data packets: the probability of loss, distortion, out of order of data packets

The Internet has been envisioned as the future platform for carrying various multimedia and computer data including video services. However, because the Internet is a best effort delivery network that does not guarantee lossless nor timely delivery, error control in video applications is a challenging research field.

Achievement of the required degree of protection and control of information is obtained by different error control methods. Error control is the process of detecting and correcting both the bit level and packet level errors. Two strategies are implemented for error control. Detection and retransmission methods such as parity check, checksum and Cyclic Redundancy Check (CRC). These methods are used to detect error and if error is detected at the receiver end then one of Automatic Repeat reQuest (ARQ) procedures may be used for the retransmission of data frame. The second strategy is forward error correction (FEC) methods using for example Hamming codes. In this method, redundant bits are included with the original data, those bits are arrange such that different incorrect bits produce different error results, that incorrect bits can be identified. Once the bit is identified, the receiver can reverse its value and correct the error. Hamming code can be applied to any length of data unit and uses the relationships between the data and the redundancy bits.

These methods are based on introducing redundancy in the transmitted data, which increases the delay time and reduces the effective data rate that leads to degradation of QoS. Introduction of redundancy in the transmitted interactive video stream limits their high speed and increase delay and jitter. 
From the above introduction we conclude the following problems:

- Any error in the transmitted interactive video stream causes severe degradation

- Repetitive ARQ may incur unacceptable delay

- FEC methods may be too complex or cause extra delay

For the solution of these problems we propos the new method which base on the following assumptions:

1. Enough bandwidth is available between source and destination

2. Multiple channels exist between source and destination

3. Independent error and failure events

4. Probability of all channels fail simultaneously is very low

5. Modification of the transmission and reception of interactive video signals

Most modern communication networks are nowadays implement fiber optics channels. This factor makes first and second assumptions realizable.

Examples of interactive two-way video communication include teleconferencing, video telephony, virtual classroom and large number of other applications. Such applications have very strong delay and jitter requirements. For effective communications, the delay and jitter must be within a certain limit. For example, for the videoconferencing, the ITU-T Recommendation P.910 standard recommends a delay of $50 \mathrm{~ms}$ for high, and $1000 \mathrm{~ms}$ for low quality (ITU-T Recommendation P.910, 99). Video classes and their attributes are presented in Table 1.

Table 1. The classes and attributes of videos

\begin{tabular}{|c|c|c|c|c|c|}
\hline $\begin{array}{l}\text { Video } \\
\text { class }\end{array}$ & $\begin{array}{l}\text { Definition and } \\
\text { Example }\end{array}$ & $\begin{array}{l}\text { Frame } \\
\text { rate (fps) }\end{array}$ & $\begin{array}{l}\text { Video bit } \\
\text { rate }(\mathrm{Mbit} / \mathrm{s})\end{array}$ & $\begin{array}{l}\text { Delay } \\
\text { ms }\end{array}$ & $\begin{array}{l}\text { Jitter } \\
\text { ms }\end{array}$ \\
\hline TV 0 & Loss-less, without compression. & 30 & 270 & $50-500$ & $50-500$ \\
\hline TV 1 & Used for complete post production & 30 & 18 to 50 & $50-500$ & $50-500$ \\
\hline TV 2 & Used for simple modifications & 30 & 10 to 25 & $50-500$ & $50-500$ \\
\hline TV 3 & $\begin{array}{l}\text { Used for delivery to home consumer, } \\
\text { cable system and high quality } \\
\text { videoconferencing }\end{array}$ & 30 & 1.5 to 8 & $50-500$ & $50-500$ \\
\hline MM 4 & $\begin{array}{l}\text { All frames encoded, medium quality } \\
\text { videoconferencing }\end{array}$ & 30 or 25 & 0.7 to 1.5 & 150 & 50 \\
\hline MM 5 & $\begin{array}{l}\text { Frames may be dropped at encoder, } \\
\text { low quality videoconferencing }\end{array}$ & $10-30$ & 0.05 to 0.2 & 1000 & 500 \\
\hline MM 6 & $\begin{array}{l}\text { Series of stills, Surveillance and } \\
\text { Graphics }\end{array}$ & Limit $\rightarrow 0$ & $<0.05$ & None & None \\
\hline
\end{tabular}

To satisfy the strong delay and jitter requirements of video applications over modern communication networks the following conditions are essentials: encoder and decoder buffers are typically very small, retransmission is usually unacceptable and Jitter in the network also has to be limited. Because of these constraints, interactive applications are considered the most demanding among different types of video communications.

The problem of error control in video communication is becoming increasingly important because of the growing interest in video delivery over unreliable channels such as wireless networks and the Internet. Several researches are found in literature. For example (Yao, 1998) reviews the techniques that have been developed for error control and concealment. In (Yunpeng, 2011) authors propose real-time feedback-based interactive error control methods suitable for low bit rate transmission.

In this paper we propose a new method of error control which differs from the proposed in (Fong, 2008; El, 2004; Fong, 2011) multi-path FEC control scheme with path interleaving for improving the quality of video transmission. The proposed scheme adaptively adjusts the FEC block size and sends interleaved data over 
multiple paths. The proposed adaptive FEC methods increase the complicity of system and retransmissions cause unacceptable delay and jitter.

The rest of the paper is organized as follows. In the next section, we describe the proposed error control method. In Section 3 we analysis and draw a model of the decision making device by deriving the functioning equation. An estimation of the probability of error and the dependence of probability of error on the number of parallel connections are described in Section 4. Finally, the results are discussed and the paper is concluded in Section 5.

\section{Error Control Method}

The proposed method is good model for the Internet and for wireless networks as video applications suffers from errors more than other communication technologies. Table 2 introduces a comparison of bit error rate (BER) for some video applications over different communication technologies (Wang, 2002).

From the above table we see the high error rate for wireless and Internet communication technologies which makes this issue very important. For this reason, we develop an error control method which associated with the following tasks:

- synthesis of the optimal decision making device at the receiver end, which realize error control of user information at the network layer

- estimate the probability of error rate at the output of decision making device

- the choice of optimal network resource to provide the required error rate level

Based on the above mentioned ideas we developed an algorithm to control error in the information stream of interactive services. In fact, the algorithm, with the help of network protocols, implementing the network between source (A) and destination (B) of information and organized by $n$ parallel connections, as shown in Figure 1. In this case, the error control procedure is performed by the decision making device at the receiving end for $n$ simultaneously received messages. First phase in algorithm based on the routing protocols that select a connection by routers (R). The second phase involves the process of establishing parallel connections with signaling protocols. At the next phase, the transmission protocols are utilized for user information containing the algorithm of the decision making device.

Table 2. BER for some applications over different communication technologies

\begin{tabular}{|c|c|c|c|c|c|}
\hline Video application & $\begin{array}{l}\text { Network } \\
\text { technology }\end{array}$ & $\begin{array}{l}\text { Video coding } \\
\text { standard }\end{array}$ & $\begin{array}{l}\text { Video } \\
\text { bit rate }\end{array}$ & $\begin{array}{l}\text { Information } \\
\text { unit size }\end{array}$ & BER \\
\hline Video on demand & ADSL & $\begin{array}{l}\text { MPEG-1 } \\
\text { MPEG-2 }\end{array}$ & $6 \mathrm{Mbps}$ & none & Error free \\
\hline \multirow[t]{2}{*}{ Videophone } & ISDN & $\begin{array}{l}\text { H-261 } \\
\text { H-263 }\end{array}$ & 64-384 kbps & none & Error free \\
\hline & PSTN & H-263 & $20 \mathrm{kbps}$ & 100 bytes & Very few \\
\hline Mobile videophone & wireless & $\mathrm{H}-263$ & 10-300 kbps & 100 bytes & $10^{-5}-10^{-3}$ \\
\hline Videophone & Internet & $\begin{array}{l}\text { H-261 } \\
\text { H-263 }\end{array}$ & $10-1000 \mathrm{kbps}$ & 1500 bytes & $0-30 \%$ \\
\hline TV broadcasting & satellite & MPEG-2 & 6-12 Mbps & 188 bytes & $\begin{array}{l}\text { Almost error } \\
\text { free }\end{array}$ \\
\hline Videoconferencing & ATM & $\mathrm{H}-262$ & 1-2 Mbps & 53 bytes & $10^{-6}-10^{-4}$ \\
\hline
\end{tabular}




\section{Synthesis of the Optimal Decision Making Device}

We assume that the source (A) generates messages $s=\left\{s_{i}\right\}, i=1,2$ with a priori probabilities of their appearance in each of $n$ parallel connection $0 \leq p\left(s_{i}\right) \leq 1 ; \quad \sum p\left(s_{i}\right)=1$. In each of the $n$ connections we allowed the modification of $S$ transmitted messages into $X=\left\{x_{1}, \ldots x_{j}, \ldots x_{n}\right\} \quad$ with independent probabilities $P_{M}^{(j)} ; j=\overline{l, n}$. Then for decision making device with $n$ parallel inputs $x_{j} ; j=\overline{1, n}$ and one output $\mathrm{Y}$ we had derived the following equations:

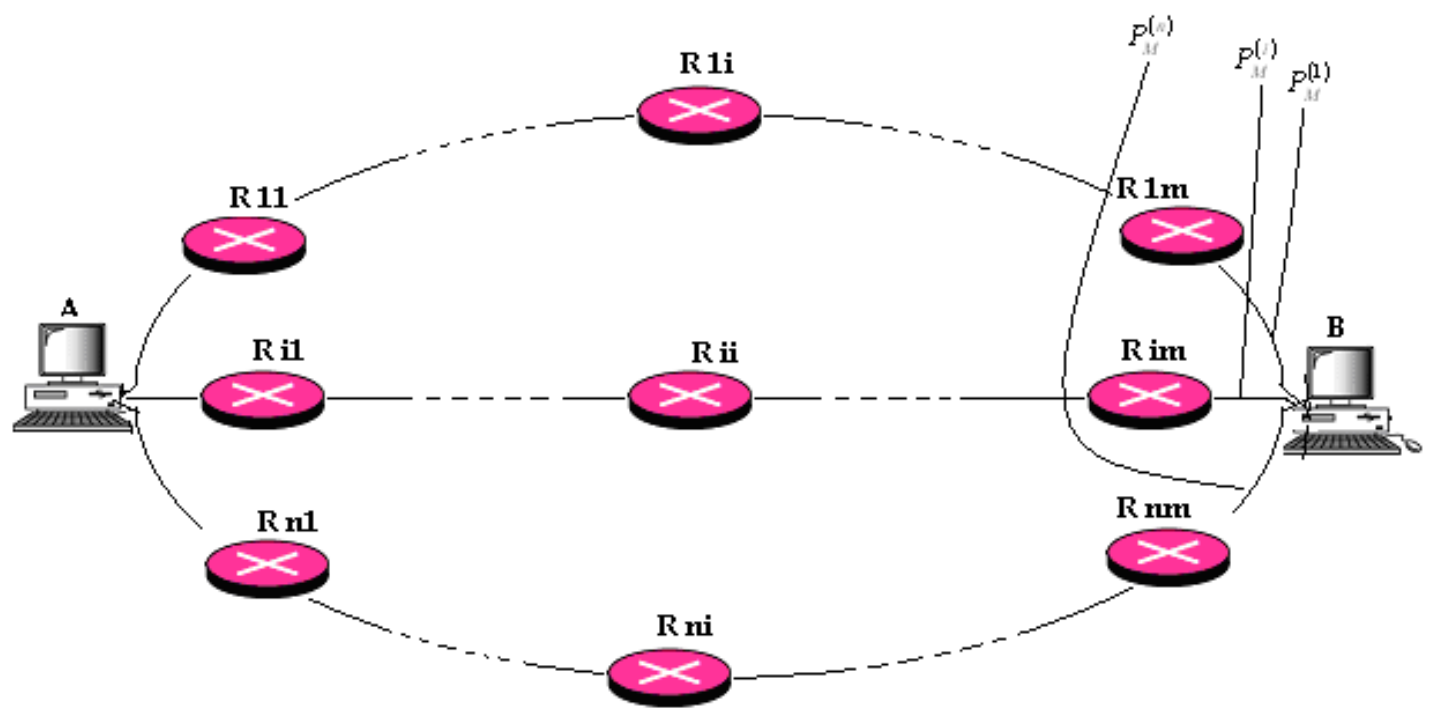

Figure 1. Conceptual model of organized $\mathrm{n}$ connections

$$
\frac{p\left\{s_{1} / x_{j} ; j=\overline{1, n}\right\}}{p\left\{s_{2} / x_{j} ; j=\overline{1, n}\right\}}=\frac{p\left(s_{1}\right)}{p\left(s_{2}\right)} \times \frac{\prod_{j \in x_{j}=s_{1}}\left(1-P_{M}^{(j)}\right)}{\prod_{j \in x_{j}=s_{l}} P_{M}^{(j)}} \times \frac{\prod_{j \in x_{j}=s_{2}} P_{M}^{(j)}}{\prod_{j \in x_{j}=s_{2}}\left(1-P_{M}^{(j)}\right)}
$$

and applying natural logarithm to both sides of Equation (1) yields

$$
\ln \frac{p\left\{s_{1} / x_{j} ; j=\overline{0, n}\right\}}{p\left\{s_{2} / x_{j} ; j=\overline{0, n}\right\}}=\ln \frac{p\left(s_{1}\right)}{p\left(s_{2}\right)}+\sum_{j \in x_{j}=s_{l}} \ln \frac{\left(1-P_{M}^{(j)}\right)}{P_{M}^{(j)}}+\sum_{j \in x_{j}=s_{2}} \ln \frac{P_{M}^{(j)}}{\left(1-P_{M}^{(j)}\right)}
$$

where $p\left\{s_{1} / x_{j} ; j=\overline{1, n}\right\}$ and $p\left\{s_{2} / x_{j} ; j=\overline{1, n}\right\}$ are conditional probability that the output signal of the 
decision making device will be $S_{1}$ or $S_{2}$.

For the analysis purposes we assume that the transmitter generates signals using NRZ line code with $s_{i}=\left\{\begin{array}{ll}+1, & \text { for } s_{1} \\ -1, & \text { for } s_{2}\end{array} s_{i}=\left\{\begin{array}{ll}+1, & \text { for } s_{1} \\ -1, & \text { for } s_{2}\end{array}\right.\right.$, then from (1) and (2)

$\ln \frac{p\left\{s_{1} / x_{j} ; j=\overline{0, n}\right\}}{p\left\{s_{2} / x_{j} ; j=\overline{0, n}\right\}}=a_{0}+\sum_{j=1} x_{j} a_{j}$,

where $a_{0}=\ln \frac{p\left(s_{1}\right)}{p\left(s_{2}\right)}$ and $a_{j}=\ln \frac{\left(1-P_{M}^{(j)}\right)}{P_{M}^{(j)}}$ are estimation coefficients.

From the derived above equations we derived the procedure of the optimal decision making device at the receiver end, which realize error control of user information at the network layer by the equation

$$
a_{0}+\sum_{j=1} x_{j} a_{j} \begin{cases}>0 & \text { then } Y=\mathrm{s}_{1} \\ <0 & \text { then } Y=\mathrm{s}_{2}\end{cases}
$$

Using this Equation (3) we can draw the block diagram of the decision making device at the receiver end which presented in Figure 2.

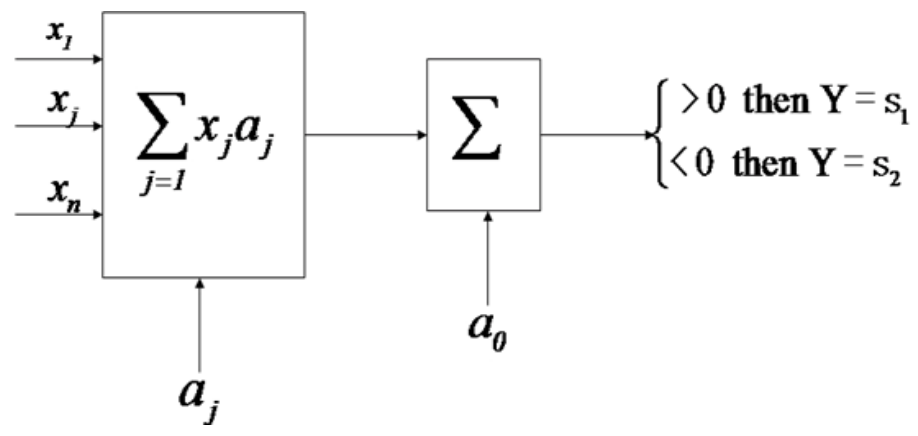

Figure 2. Block diagram of the decision making device

\section{Estimation of Probability of Error}

Let the probabilities of modifying over the $n$ inputs of the decision making device are independent and equal that

$$
P_{M}=P_{M}^{(j)} ; j=\overline{1, n}
$$

Then the probability of erroneous decision at the output of the decision making device is given as

$$
P_{\text {error }}=\sum_{j=0}^{\frac{n-1}{2}} c_{n}^{\frac{n+1+2 j}{2}}\left(1-P_{M}\right)^{\frac{n-1-2 j}{2}} P_{M}^{\frac{n+1+2 j}{2}}
$$

For the presentation of the probability of erroneous decision calculated by Equation (4) we used the simulation program MatLab and obtained the result curves in Figure 3. The calculation is done for different values of $n$, and using the function $P_{\text {error }}=f\left(X, \delta_{X}\right)$, where $\delta_{n}$ represents the expectation factor. 


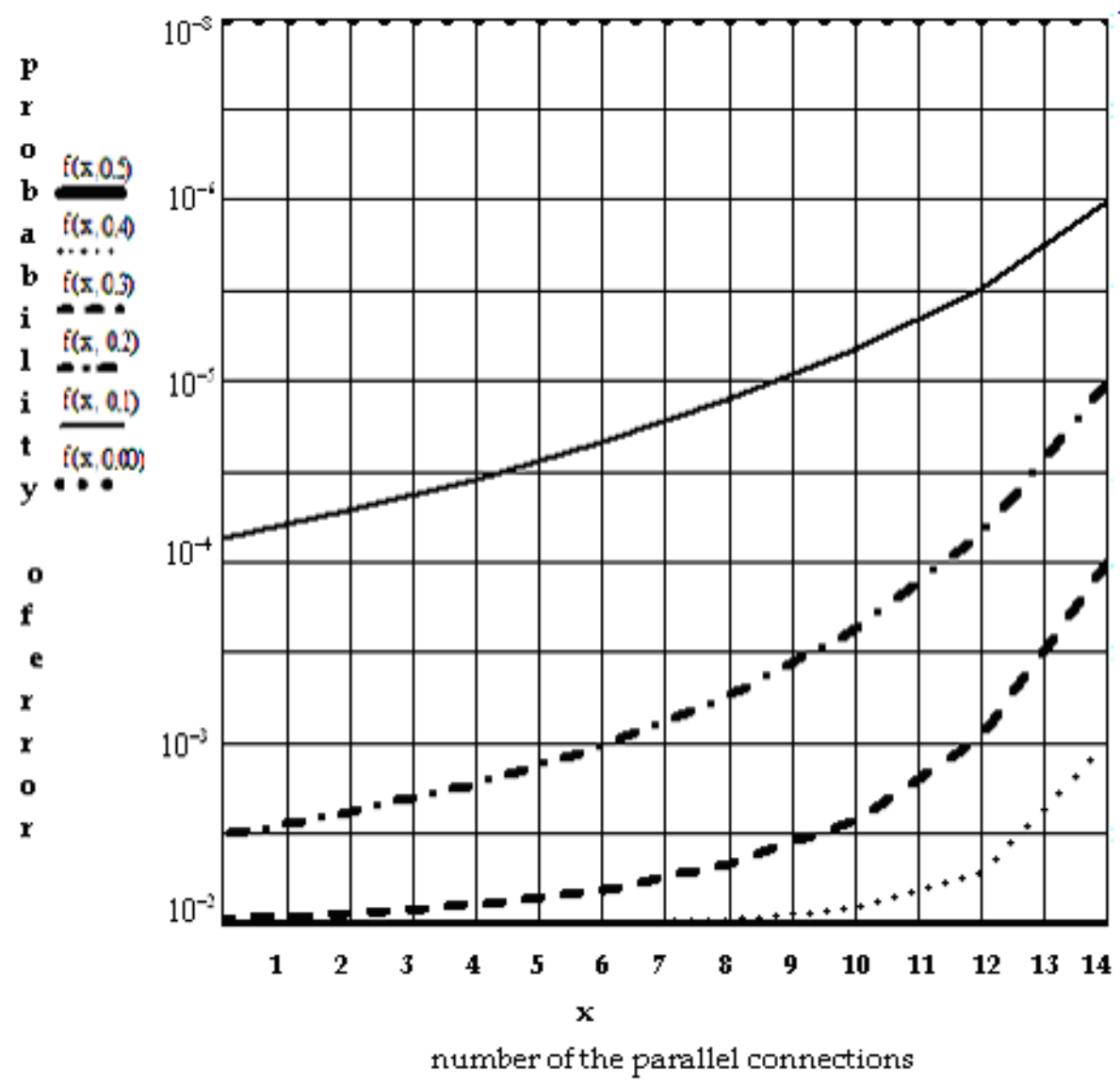

Figure 3. The dependence of probability of error on the number of parallel connections

\section{Discussion and Conclusion}

From Figure 3 we clearly note the following:

1. As the number of parallel connections $\mathrm{x}$ increased as the probability of error decrease; it achieves $10^{-6}$, which means that one bit may be erroneously received for $10^{6}$ bits transmitted, when $\mathrm{x}=14$ and $\delta_{X}=0.1$.

2. We found the BER improvement achieved by $\mathrm{x}=14$ and $\delta_{X}=0.1$ which is $10^{-6}$, this value is less than the BER provided in Table 2 for mobile videophone over wireless technology and over Internet.

3. As the expectation factor increase as the probability of error increased; for example, when we take $x=10$ and compare the resulting probability of error for different $\delta_{X}=0.2$ and $\delta_{X}=0.4$ we find the probability of error equal $10^{-3.6}$ and $10^{-2.1}$ respectively.

\section{References}

El, A. A., Saadawi, T., \& Lee, M. (2004). A multi-path error control mechanism for interactive video in mobile wireless networks. Applications and Services in Wireless Networks, ASWN, 161-168.

ITU-T Recommendation P.910 (09/99) Subjective video quality assessment methods for multimedia applications.

Liu, Y., Zhang, S., Zhang, Y., Wang, R., \& Li, J. (2011). Interactive H.264/SVC error control methods design and implementation. International Conference on Image Analysis and Signal Processing (IASP), 424-428.

Tsai, M. F., Chilamkurti, N., \& Shieh, C. K. (2008). A novel multi-path forward error correction control scheme with path interleaving for video transmissions. Telecommunications, ICT, 1-8.

Tsai, M. F., Chilamkurti, N. K., Zeadally, S., \& Vinel, A. (2011). Concurrent multi-path transmission combining forward error correction and path interleaving for video streaming. Computer Communications, 34(7), 
1125-1136. http://dx.doi.org/10.1016/j.comcom.2010.02.001

Wang, Y., Ostermann, J., \& Zhang, Y. Q. (2002). Video processing and communications. Prentice-Hall, ISBN.

Wang, Y., \& Zhu, Q. F. (1998). Error control and concealment for video communication: A review. Proceedings of the IEEE, 86, 974-997. http://dx.doi.org/10.1109/5.664283 